Review Article

\title{
Research Progress in Fecal Microbiota Transplantation as Treatment for Irritable Bowel Syndrome
}

\author{
Yao Wang $\mathbb{D},{ }^{1}$ Fengling Zheng, ${ }^{2}$ Shan Liu, ${ }^{1}$ and Huanhuan Luo $\mathbb{D}^{1}$ \\ ${ }^{1}$ School of Basic Medicine, Guangzhou University of Chinese Medicine, Guangzhou, Guangdong Province 510006, China \\ ${ }^{2}$ The Journal Center, Guangzhou University of Chinese Medicine, Guangzhou, Guangdong Province 510006, China
}

Correspondence should be addressed to Huanhuan Luo; avenluo@gzucm.edu.cn

Received 17 April 2019; Revised 10 September 2019; Accepted 7 November 2019; Published 1 December 2019

Academic Editor: Per Hellström

Copyright (c) 2019 Yao Wang et al. This is an open access article distributed under the Creative Commons Attribution License, which permits unrestricted use, distribution, and reproduction in any medium, provided the original work is properly cited.

Irritable bowel syndrome is a functional disorder characterized by abdominal pain or discomfort associated with altered bowel habits. Due to the uncertainty of the pathogenesis of IBS and the diversity of its clinical manifestations, IBS cannot be completely cured. Increasing evidence suggests the key role of altered intestinal microbiota in the pathogenesis of IBS. Therefore, attention is being shifted to adjusting the changes in intestinal microbiota to control IBS symptoms. Fecal microbiota transplantation (FMT), antibiotics, probiotics, and synbiotics are currently often employed as treatment for IBS. And FMT is the most significant therapeutic efficacy with the least number of side effects. FMT provides a creative way to restore the abnormal gut microbiome in patients with IBS. But although current clinical studies confirm the effectiveness of FMT in the treatment of IBS, they are short-term studies of small samples, and there is still a lack of large-scale long-term studies. In this paper, we review the intestinal microbiota changes of IBS, the common methods of treating IBS with intestinal microbes, and the research status of FMT for the treatment of IBS. Finally, we put forward some opinions on the future research direction of FMT treatment of IBS.

\section{Introduction}

Approximately 100 trillion different microorganisms inhabit the human intestine [1]. The gastrointestinal tract harbors the largest microbial population in the human body, accounting for $80 \%$ of the total microbial biomass. Nearly 1,014 bacteria dominate the microbiota in the gastrointestinal tract, with the genome of the intestinal microbiota having been estimated to contain almost 100 times more genes than the human genome $[2,3]$. In addition to bacteria, gut microbes include fungi, fields, viruses, and eukaryotes [4]. And the degree of parasitic infection may be related to differences in the gut microbiome [5]. The composition of the intestinal microbiota varies from one individual to another, and studies have shown that the intestinal microbiota plays a crucial role in human health and diseases via its involvement in the regulation of immune and metabolic functions [6-9]. Dysbiosis, defined as a microbial imbalance in the body [10], is an important cause of digestive tract diseases. Intestinal dysbiosis can lead to inflammatory bowel disease [11], chronic fatigue syndrome [12], obesity [13], cancer [14], metabolic diseases [15], and irritable bowel syndrome (IBS). In particular, IBS is a functional disorder characterized by abdominal pain or discomfort associated with altered bowel habits [16]. IBS diminishes patients' quality of life, resulting in psychological problems such as anxiety and depression [17]. Although IBS is not fatal [18], failure to treat IBS can lead to impairment in quality of life among IBS patients. Currently, IBS is mainly diagnosed based on medical history and physical signs, with the aid of endoscopic and imaging examinations to exclude organic diseases. According to the Rome IV diagnostic criteria for IBS [19], which were launched at the "Ninth Annual American Gastroenterological Association-Rome Foundation Lectureship" held at the 2016 Digestive Disease Week meeting, IBS can be categorized into the following four subtypes based on predominant bowel habits: IBS with predominant constipation (IBS-C), IBS with predominant diarrhea (IBS-D), IBS with mixed bowel habits (IBS-M), and unclassified IBS (IBS-U) [20]. Despite the availability of numerous drugs for the 
control of IBS symptoms, several patients do not respond well to these drugs [21]. The uncertainty about the pathogenesis of IBS and the diversity of its clinical manifestations present considerable difficulties for IBS treatment. An increasing body of evidence has confirmed that IBS is closely related to intestinal dysbiosis. Patients with different types of IBS develop diverse disorders associated with the microbiota and exhibit changes in the number and types of microbiota [22]. Furthermore, IBS patients have been reported to have a smaller number of intestinal Bifidobacterium species but a larger number of Escherichia coli [23]. The distal colonic mucosa in IBS-C patients contains fewer aerobic bacteria than that in healthy individuals [24]. IBS-D patients have a decreased number of Bacteroides, Bifidobacterium, and Ruminococcus species [25]. In comparison, IBS-M patients have a significantly decreased number of Lactobacillus species and an increased number of Enterobacteriaceae species in their feces and exhibit lower resistance to intestinal colonization than healthy individuals [26]. Lactulose hydrogen breath test can verify the occurrence of small intestinal bacterial overgrowth (SIBO) at varying degrees in IBS patients [25]. Therefore, attention is being shifted to adjusting the changes in intestinal microbiota in order to control IBS symptoms. Clinical research has begun to explore IBS treatment targeting the intestinal microbiota. Fecal microbiota transplantation (FMT), antibiotics, probiotics, and synbiotics [27] are currently often employed as treatment for IBS, with FMT showing the most significant therapeutic efficacy with the least number of side effects. FMT refers to the transplantation of a fecal matter solution from a donor into the gastrointestinal tract of a patient in order to directly change the recipient's microbial composition and hence treat the disease $[28,29]$. An increasing number of clinical studies have investigated IBS treatment using FMT; nonetheless, there exist few reviews in this regard. Therefore, this paper is aimed at reviewing the progress and shortcomings in the use of FMT as treatment for IBS based on existing clinical studies so as to improve the application of FMT to IBS treatment.

\section{Pathogenesis of IBS}

IBS is a multifactorial disease with a complex pathogenesis and pathophysiological mechanisms. Current research suggests that the pathogenesis of IBS is related to the interaction between the brain-gut axis, immune system, and intestinal microecology [30]. PI-IBS patients exhibit increased peripheral blood levels of tumor necrosis factor- $\alpha$, interleukin-1, and interleukin-6, with observable CD3 and CD25 cells and lymphocytes in the intestinal mucosa [31]. The underlying mechanisms responsible for PI-IBS remain unclear and may involve residual inflammation or persistent changes in mucosal immune cells, enterochromaffin-like cells, mast cells, intestinal nerves, and gastrointestinal microbes [32]. A number of research groups have attempted to evaluate the effects of the microbiome on the brain-gut axis. The braingut axis is a bidirectional communication pathway deemed essential for homeostasis maintenance, and part of the brain-gut axis causes important pathophysiological effects in regulatory disorders; that is, changes in brain-gut interactions have a certain effect on intestinal inflammation and abdominal pain symptoms [33]. The pathogenesis of IBS cannot be explained by a single mechanism, and changes in the intestinal flora have become the focus of concern [34].

\section{Intestinal Microbiota and IBS}

Microorganisms help maintain the human body's normal physiological functions $[35,36]$. The intestine harbors approximately 1,150 species [2], which mainly include anaerobic bacteria such as Bacteroides, Bifidobacterium, Actinomyces, Firmicutes, and Proteobacteria species. The genome of the intestinal microbiota contains almost 100 times more genes than the human genome [3]. It is generally believed that the pathogenesis of IBS is related to the intestinal barrier function, central and splanchnic neurological changes, mental and psychological stress, microinflammation, and intestinal dysmotility [4]. Recent studies have reported that intestinal dysbiosis in IBS patients may be the initiating factor in the pathogenesis of IBS [37]. The human intestinal microbiota is an extremely complex and metabolically active microbial ecosystem [34] involved in the development of the host's immune system [38, 39], maintenance of normal physiological functions of the digestive system [40], and fermentation of undigested carbohydrates [41]. The intestinal microbiota comprises three types of bacteria-namely, symbiotic bacteria, conditionally pathogenic bacteria, and transient bacteria. The classic findings to confirm the bacteria in IBS might be using rifaximin in IBS-D by RCT studies [42]. Symbiotic bacteria are important for healthy digestion in that they produce enzymes and metabolites that help the body absorb essential nutrients and vitamins [43]. Conditionally, pathogenic bacteria are invasive under certain conditions, whereas transient bacteria can cause disease when dysbiosis occurs and their number exceeds the normal level. The physiological functions of the intestinal microbiota can be primarily summarized into the following five aspects: (1) regulation of mucosal immune responses in the gastrointestinal tract; (2) activation of immune factors, strengthening of immune functions, and enhancement of disease resistance; (3) promotion of immune organs' growth and development and initiation of immune response; (4) creation of a bacterial barrier; and (5) interference with cellular immunity and stimulating the body to produce an immune response when existing as probiotics. Under normal circumstances, the intestinal microbiota can maintain a dynamic balance to promote bodily health. However, intestinal dysbiosis can induce or aggravate diseases [44] such as metabolic syndrome, IBS, and inflammatory bowel disease and is associated with some extraintestinal diseases $[45,46]$. In addition, there are some studies on fungi, parasites, and viruses in intestinal microbes that are also involved in the development of IBS. In addition, some studies have shown that fungi, parasites, and viruses in gut microbes are also associated with the occurrence of IBS. For example, S. boulardii can reduce the number of intestinal peristalsis in patients with irritable bowel syndrome and diarrhea (IBS-D) [47]. A case-control control 
study has shown the infection rate of intestinal parasites in patients with IBS which is not high compared to healthy people [48]. Nourrisson et al. showed that the level of intestinal Bifidobacterium sp. in male IBS-C patients was lower than in the normal control group. No studies have clarified which specific microbes are particularly relevant to IBS [49], and healthy populations and patients with severe or mild/moderate IBS can be identified based on the structural features of the intestinal microbiota [6]. Microbial diversity in fecal samples from IBS-D patients is significantly reduced [50], with the intestinal microbiota mainly characterized by an increased number of Enterobacteriaceae species and a decreased number of Lactobacillus, Bifidobacterium, and Bacteroides species [51]. The intestinal microecology in IBS-C patients is characterized by an increased number of Firmicutes and Bacteroidetes species [52], with constipation resulting from excessive methane production [53]. In comparison, IBS-M patients show a significantly increased number of Enterobacteriaceae species in their feces, significantly decreased number of Lactobacillus species, reduced resistance to intestinal colonization, and increased abundance of Bacteroidetes species [53, 54]. Changes in the intestinal microbiota in IBS-U patients remain unclear.

The severity of IBS symptoms is inversely correlated with microbial richness and unique microbial markers [55]. For instance, Bifidobacteria are inversely associated with pain [56], and both Bifidobacteria and Lactobacilli are inversely associated with defecation frequency [53]. In addition, cyanobacteria are associated with satiety, bloating, and gastrointestinal symptoms, whereas proteobacteria are associated with psychological and pain thresholds [57]. Ruminococcus species are associated with the severity of IBS symptoms [58]. IBS patients mainly show a decrease in the number of dominant intestinal microbiota, intestinal microbial diversity, stability of intestinal microecology, and an abundance of Bifidobacteria and Lactobacilli, along with an increase in the number of enterobacteria and a decline in resistance to intestinal colonization. Patients with different types of IBS have diverse changes in the intestinal microbiota. In clinics, it is possible to conduct targeted treatment according to changes in the intestinal microbiota. Promoting intestinal microbial diversity and restoring the balance of the intestinal microbiota are particularly important in IBS treatment.

\section{Application of Interventions Targeting the Intestinal Microbiota to IBS Treatment}

Currently, the use of fermentable oligosaccharides, disaccharides, monosaccharides, and polyols (FODMAP), antibiotics, and probiotics as treatment for IBS has achieved a certain level of therapeutic efficacy. Commonly found in food, FODMAP are short-chain carbohydrates that are not readily absorbed in the small intestine. A low-FODMAP diet for 3 weeks has been shown to significantly improve IBS symptoms, increase the abundance and diversity of Actinomyces in the fecal microbiota, and decrease the urinary concentration of histamine [59]. It is currently accepted that SIBO is most closely related to IBS, prompting the proposal that anti- biotics could be used as treatment for IBS [60]. Pistiki et al. [61] isolated 170 types of aerobic bacteria from the intestines of patients diagnosed with SIBO-induced IBS.

Furthermore, they reported that the in vitro use of $32 \mu \mathrm{g} / \mathrm{mL}$ rifaximin inhibited $85.4 \%$ of E. coli, $43.6 \%$ of Klebsiella species, $34.8 \%$ of Enterobacteriaceae species, $54.5 \%$ of other Enterobacteriaceae species, $82.6 \%$ of Gram-negative non-Enterobacteriaceae species, and 100\% of Enterococcus faecalis and Staphylococcus aureus, suggesting the antibacterial effect of rifaximin on small intestinal bacteria related to SIBO. In addition, patients with nonconstipation IBS showed improvement in bloating, abdominal pain, and fecal traits after treatment with rifaximin [62], and 5-HT3 receptor antagonists can improve symptoms in IBS-D patients [63]. The mechanism by which antibiotics treat IBS may be related to the changes in the ratio of intestinal microbiota and prevention of intestinal inflammation and visceral hypersensitivity due to stress [64].

Probiotics are living microorganisms that are beneficial to the human body [65]. Probiotics can restore the ecological balance of the intestinal microbiota and alter the expression and redistribution of tight junction proteins, can restrict the absorption of harmful molecules in the intestinal lumen by reducing intestinal mucosal permeability, and can affect the inflammatory response of epithelial cells to the stimuli in the intestinal lumen by secreting various factors, thereby reducing mucosal inflammation while acquiring immunity by affecting mucosal T-cells in the lamina propria $[66,67]$. Probiotics can improve abdominal pain symptoms in IBS patients and alleviate overall symptoms but are not effective in improving the symptoms of bloating, constipation, and diarrhea [68]. Supplementation with Lactobacilli or Bifidobacteria not only reduces the intestinal mucosal inflammatory response in PI-IBS patients [69] but also significantly improves the symptoms of diarrhea and constipation [70]. A study by Johnsen et al. showed that FMT could significantly reduce the symptoms of IBS patients but did not affect the patients' microbiome [71]. According to a 2018 study, the microbiome of IBS patients was closely similar to that of donors after FMT capsule treatment, and FMT capsules had a significant effect on the recipients' microbiome [72]. Methods for FMT include the use of nasogastric or nasojejunal tube and endoscopic approaches, with the colonoscopic approach showing the best performance. The recent metaanalysis shows FMT treatment CDI (Clostridium difficile infection) has a good effect [73].

\section{Current Status of FMT}

5.1. Donor Selection. As is the case with other treatments, FMT poses some risk. Despite the absence of a uniform standard for "qualified" microbial communities, careful selection of FMT donors is necessary. Only a healthy individual without autoimmune or metabolic disease, family history of malignant disease, or any potential pathogen can become a fecal donor [74]. Although patients are allowed to choose relatives as the source of transplanted feces in early FMT $[75,76]$, there exist no definitive statistical data confirming that selecting relatives as donors would improve the quality 
of FMT. Irrespective of the type of donor selected for FMT, each donor should be carefully and thoroughly screened prior to FMT [77-79]. Different patients and donors are subject to individualized adjustments and require additional examinations [80]. Currently, the prevailing screening method is to ensure-as far as possible-the absence of diseases due to intestinal microbes, blood-related disorders, or bacterial or parasitic infections transmitted through FMT while fulfilling the inclusion criteria.

5.2. Methods for the Preparation of Mixed Liquid. Currently, the main preparation method involves repeatedly weighing, preparing, filtering, and centrifuging the fecal liquid in order to produce a mixture of fecal bacteria. This conventional preparation method is time-consuming, labor-intensive, and inefficient, failing to meet clinical requirements. Cui et al. [81, 82] developed a smart microbiota treatment system that standardizes the procedure of precise fecal bacterial processing, multistage filtration, repeated centrifugation, concentration, and enrichment. Because of rapid preparation, which minimizes air contact, a large number of anaerobic bacteria in the fecal liquid are allowed to survive to the greatest extent, thus making the prepared fecal microbiota basically similar to the original fecal microbiota with respect to structural composition. Studies have shown that suspension prepared with water has a higher resolution than that prepared with physiological saline, although the recurrence rate of the former is higher; in addition, yogurt, milk, or psyllium-containing brine [83] can be used as a diluent. Nonetheless, some precautions must be taken: (1) In order to avoid mass reproduction of aerobic bacteria, the preparation process should be simple as much as possible [84]. (2) After processing, the mixture can be directly injected into the gastrointestinal tract or can be prepared as capsules for administration [79]. (3) Lyophilized capsules are small in volume and can be stored for a long time [85]. (4) A case report in 2012 indicated that the use of standardized frozen feces was not statistically different from the use of fresh feces [86].

5.3. Route of Administration for FMT. The fecal liquid can be injected into the intestine through a variety of ways, mainly via nasogastric tubes, nasojejunal tubes, upper gastrointestinal endoscopes, colonoscopes, or retention enema. Colonoscopy enables the lesion to be directly observed; however, being an invasive examination, colonoscopy may cause damage to the colon and is costly [84]. Although the use of enema is less costly and invasive than the colonoscopic approach, its performance is less satisfactory owing to the limited range of intestinal sites that the enema can reach [84]. The fecal liquid can also be injected via the upper digestive tract, which is simple and has few complications. Only a limited number of studies have explored the effect of administration route for FMT on clinical efficacy, and a large number of clinical trials are required for verification. The optimal administration route may depend on the location of the lesion, characteristics of the disease, and general condition of the patient. No consensus on the frequency of FMT has been achieved yet, and FMT may be performed either multiple times (inter- mittent or continuous administration) or as a single administration [79]. Each route has its own advantages and disadvantages, and the appropriate route should be selected based on the characteristics of the disease. For instance, in the case of abdominal diseases related to the small intestine, FMT is recommended to be performed via the duodenum. Crohn's disease often affects the entire gastrointestinal tract, preferentially the distal ileum and right hemicolon. Therefore, the upper gastrointestinal route may be more appropriate. Using the colonoscopic route in patients with severe colonic inflammation or colonic flatulence may entail a high risk; hence, the upper gastrointestinal route can be adopted instead [87]. The administration route should be determined according to the specific conditions of the patient.

\section{Clinical Efficacy of FMT in Treating IBS}

Probiotics can restore the intestinal microecology in IBS patients. Dissimilar to probiotics containing a small number of bacterial strains, FMT feces includes almost all bacteria from healthy donors. To some extent, fecal bacteria are the ultimate probiotics in humans [88]. In 1989, McEvoy [89] was the first to use FMT as treatment for IBS and inflammatory bowel disease, with a cure rate of 36\% (20/55). Pinn et al. [90] employed FMT to treat refractory IBS patients (9 IBS-D patients, 3 IBS-C patients, and 1 IBS-M patient) and reported an overall remission rate of $70 \%$ for IBS, including improvement in abdominal pain (72\%), bowel habits (69\%), indigestion (67\%), bloating (50\%), and venting (42\%). Moreover, Mazzawi et al. [91] explored the effects of FMT on symptoms and duodenal enteroendocrine cells in IBS patients and reported the effectiveness of FMT in treating IBS-D patients, revealing that IBS symptom scores for abdominal pain $(P=0.005)$, diarrhea $(P=0.004)$, and anorexia $(P=0.096)$; IBS severity scores $(P=0.0002)$; and Bristol Stool Scale scores $(P=0.02)$ were significantly decreased after FMT treatment for 3 weeks. Gaidar et al. [92] investigated the effectiveness of colonoscopy-assisted FMT in treating refractory IBS-D, IBS-C, and IBS-M patients. They reported that $75 \%$ of patients experienced relief from abdominal pain $(P<0.01)$ and that stool frequency and consistency in all IBS-M and IBS-D patients returned to normal. In 2017, the first randomized controlled trial (RCT) on FMT treatment for IBS [93] was conducted in Norway, primarily enrolling severe IBS patients who met the Rome III criteria. The patients were assigned to a group $(n=60)$ comprising subjects who received $50-80 \mathrm{~g}$ of fresh FMT (used on the same day) or frozen FMT and a group $(n=30)$ consisting of subjects who received his or her own feces as placebo. Transplantation was performed with colonoscopy. After 3 months of FMT treatment, the Irritable Bowel Syndrome Symptom Severity Scale scores decreased by more than 75 points in 36 out of 55 subjects who were actively treated $(65 \%)$ and 12 out of 28 subjects who received placebo $(43 \%)(P=0.049)$, indicating that therapeutic efficacy was significantly better in the treatment group than in the placebo group. In 2018, Holvoet et al. [94] performed an RCT that included patients who were diagnosed with Rome III IBS complicated with severe bloating but did not have constipation. A total of 64 patients were 
allocated to the fresh FMT group, with each subject treated with either frozen feces from two donors or the subject's own feces. Transplantation was performed using an electromagnetically guided nasojejunal tube. At week 12, 49 FMT recipients showed adequate relief from IBS symptoms and bloating, compared with 29 in the control group $(P=0.004)$. In a recent RCT conducted by Johnsen et al., the IBS severity score of patients who received donor FMT via colonoscopy decreased, as compared with that of patients who received autologous FMT [71]. Nevertheless, this RCT showed that the effect of autogenous feces transported via colonoscopy or nasal intestinal tubes was better than that of donor feces $[71,95]$. Some studies reported that FMT has no significant effect on IBS treatment. The results of the RCT indicated that when FMT was administered via capsules, placebo capsules were better than capsules containing donor feces [72, 96, 97]. The placebo effect is common in clinical trials on IBS [98], which may be related to IBS itself being a functional bowel disease [99]. Thus far, trials on FMT-based treatment for IBS have been mostly conducted with small sample sizes and lack long-term follow-up. The relationship between gut microbes and IBS remains not entirely clear, which may be the reason for the ineffective FMT treatment. Furthermore, the relationship between intestinal microbes and IBS is not fully understood, which may explain why FMT is not effective in treating some IBS patients. Adverse reactions to FMT treatment should also attract our attention; most of these adverse reactions are gastrointestinal symptoms, most patients experience transient diarrhea after FMT treatment, and a few may manifest symptoms such as bloating and belching, which usually disappear after 2-3 days [85]. Additionally, studies reported that adverse reactions, including death $(0.25 \%)$, perforation/tear $(0.25 \%)$, and Gram-negative bacteremia $(0.34 \%)$, might occur in CDI patients receiving FMT; although the incidence is not high, we should consider it important because of the threat that adverse reactions constitute to health [100]. Owing to a short follow-up period, it is not possible to assess long-term efficacy or the occurrence of an underlying disease. Therefore, high-quality RCTs with large samples and long-term follow-up are required to further investigate the therapeutic effect of FMT on IBS.

\section{Conclusions}

Although FMT for CDI has a significant effect, its effectiveness in treating IBS needs to be further explored. Reconstructing a healthy microecology is desirable, but feces contain not only bacteria but also viruses, phages, fungi, protozoa, cells, mucus, enzymes, and metabolites-all of which affect the entire human ecosystem. In future research, the underlying mechanism of microbiome-host interaction and the role of microbiome in the pathogenesis of the disease should be elucidated. To this end, it is necessary to explore the specificity of the intestinal microbiota in each IBS subtype (i.e., specifying which bacteria are associated with which symptoms); by doing so, we may identify a different treatment approach. Moreover, exploring whether FMT restores a patient's intestinal microecology or the functions of some microbes is recommended. It is possible to further clarify the changes in the structural composition of the intestinal microbiota and the difference in intestinal microbiota between the patient and the donor before and after FMT treatment by employing metagenomic detection technology.

\section{Conflicts of Interest}

The authors declare that there is no conflict of interests regarding the publication of this paper.

\section{References}

[1] K. Honda and D. R. Littman, "The microbiome in infectious disease and inflammation," Annual Review of Immunology, vol. 30, no. 1, pp. 759-795, 2012.

[2] J. Qin, M. H. I. T. Consortium, R. Li et al., "A human gut microbial gene catalogue established by metagenomic sequencing," Nature, vol. 464, no. 7285, pp. 59-65, 2010.

[3] R. E. Ley, D. A. Peterson, and J. I. Gordon, "Ecological and evolutionary forces shaping microbial diversity in the human intestine," Cell, vol. 124, no. 4, pp. 837-848, 2006.

[4] C. A. Lozupone, J. I. Stombaugh, J. I. Gordon, J. K. Jansson, and R. Knight, "Diversity, stability and resilience of the human gut microbiota," Nature, vol. 489, no. 7415, pp. 220-230, 2012.

[5] C. R. Stensvold and M. van der Giezen, "Associations between gut microbiota and common luminal intestinal parasites," Trends in Parasitology, vol. 34, no. 5, pp. 369377, 2018.

[6] J. Tap, M. Derrien, H. Törnblom et al., "Identification of an intestinal microbiota signature associated with severity of irritable bowel syndrome," Gastroenterology, vol. 152, no. 1, pp. 111-123.e8, 2017.

[7] The Human Microbiome Project Consortium, "Structure, function and diversity of the healthy human microbiome," Nature, vol. 486, no. 7402, pp. 207-214, 2012.

[8] S. V. Lynch and O. Pedersen, "The human intestinal microbiome in health and disease," The New England Journal of Medicine, vol. 375, no. 24, pp. 2369-2379, 2016.

[9] A. B. Hall, A. C. Tolonen, and R. J. Xavier, "Human genetic variation and the gut microbiome in disease," Nature Reviews Genetics, vol. 18, no. 11, pp. 690-699, 2017.

[10] C. P. Tamboli, C. Neut, P. Desreumaux, and J. F. Colombel, "Dysbiosis in inflammatory bowel disease," Gut, vol. 53, no. 1, pp. 1-4, 2004.

[11] P. Marteau, "Bacterial flora in inflammatory bowel disease," Digestive Diseases, vol. 27, no. 1, pp. 99-103, 2009.

[12] S. E. Lakhan and A. Kirchgessner, "Gut inflammation in chronic fatigue syndrome," Nutrition \& Metabolism, vol. 7, no. 1, p. 79,2010

[13] P. J. Turnbaugh, R. E. Ley, M. A. Mahowald, V. Magrini, E. R. Mardis, and J. I. Gordon, "An obesity-associated gut microbiome with increased capacity for energy harvest," Nature, vol. 444, no. 7122, pp. 1027-1031, 2006.

[14] M. Castellarin, R. L. Warren, J. D. Freeman et al., "Fusobacterium nucleatum infection is prevalent in human colorectal carcinoma," Genome Research, vol. 22, no. 2, pp. 299-306, 2012. 
[15] K. Mazmanian Sarkis, "Capsular polysaccharides of symbiotic bacteria modulate immune responses during experimental colitis," Journal of Pediatric Gastroenterology and Nutrition, vol. 46, Suppl 1, pp. E11-E12, 2008.

[16] R. M. Lovell and A. C. Ford, "Global prevalence of and risk factors for irritable bowel syndrome: a meta-analysis," Clinical Gastroenterology and Hepatology, vol. 10, no. 7, pp. 712-721.e4, 2012.

[17] M. A. Malone, "Irritable bowel syndrome," Primary Care: Clinics in Office Practice, vol. 38, no. 3, pp. 433-447, 2011.

[18] P. Paré, J. Gray, S. Lam et al., "Health-related quality of life, work productivity, and health care resource utilization of subjects with irritable bowel syndrome: baseline results from LOGIC (Longitudinal Outcomes Study of Gastrointestinal Symptoms in Canada), a naturalistic study," Clinical Therapeutics, vol. 28, no. 10, pp. 1726-1735, 2006, discussion $1710-1$.

[19] D. A. Drossman, "Functional gastrointestinal disorders: history, pathophysiology, clinical features, and Rome IV," Gastroenterology, vol. 150, no. 6, pp. 1262-1279.e2, 2016.

[20] G. F. Longstreth, W. G. Thompson, W. D. Chey, L. A. Houghton, F. Mearin, and R. C. Spiller, "Functional bowel disorders," Gastroenterology, vol. 130, no. 5, pp. 1480-1491, 2006.

[21] W. D. Chey, J. Kurlander, and S. Eswaran, "Irritable bowel syndrome: a clinical review," JAMA, vol. 313, no. 9, pp. 949-958, 2015.

[22] E. M. M. Quigley, H. Abdel-Hamid, G. Barbara et al., "A global perspective on irritable bowel syndrome: a consensus statement of the World Gastroenterology Organisation Summit Task Force on irritable bowel syndrome," Journal of Clinical Gastroenterology, vol. 46, no. 5, pp. 356-366, 2012.

[23] J.-M. Si, Y.-C. Yu, Y.-J. Fan, and S.-J. Chen, "Intestinal microecology and quality of life in irritable bowel syndrome patients," World Journal of Gastroenterology, vol. 10, no. 12, pp. 1802-1805, 2004.

[24] G. Barbara, V. Stanghellini, C. Berti-Ceroni et al., "Role of antibiotic therapy on long-term germ excretion in faeces and digestive symptoms after Salmonella infection," Alimentary Pharmacology and Therapeutics, vol. 14, no. 9, pp. 1127$1131,2000$.

[25] A. Kassinen, L. Krogius-Kurikka, H. Mäkivuokko et al., “The fecal microbiota of irritable bowel syndrome patients differs significantly from that of healthy subjects," Gastroenterology, vol. 133, no. 1, pp. 24-33, 2007.

[26] Y. Bo, L. Cheng, and Z. Xuchun, "Effects of changes of interstitial cells of cajal from mice with post-infection irritable bowel syndrome on intestinal motility and visceral sensitivity," Journal of Shanghai Jiaotong University, vol. 34, no. 7, pp. 978-983, 2014.

[27] S.-H. Lee, D.-Y. Cho, S.-H. Lee et al., “A randomized clinical trial of synbiotics in irritable bowel syndrome: dosedependent effects on gastrointestinal symptoms and fatigue," Korean Journal of Family Medicine, vol. 40, no. 1, pp. 2-8, 2019.

[28] J. S. Bakken, T. Borody, L. J. Brandt et al., "Treating Clostridium difficile infection with fecal microbiota transplantation," Clinical Gastroenterology and Hepatology, vol. 9, no. 12, pp. 1044-1049, 2011.

[29] L. P. Smits, K. E. C. Bouter, W. M. De Vos, T. J. Borody, and M. Nieuwdorp, "Therapeutic potential of fecal microbiota transplantation," Gastroenterology, vol. 145, no. 5, pp. $946-$ 953, 2013.

[30] T. Borody, J. Campbell, M. Torres, A. Nowak, and S. Leis, "Reversal of idiopathic thrombocytopenic purpura [itp] with fecal microbiota transplantation [FMT]," The American Journal of Gastroenterology, vol. 106, p. S352, 2011.

[31] R. Spiller and C. Lam, "An update on post-infectious irritable bowel syndrome: role of genetics, immune activation, serotonin and altered microbiome," Journal of Neurogastroenterology and Motility, vol. 18, no. 3, pp. 258-268, 2012.

[32] R. Spiller and K. Garsed, "Postinfectious irritable bowel syndrome," Gastroenterology, vol. 136, no. 6, pp. 1979-1988, 2009.

[33] M. R. B. Keighley, "Emergency abdominal surgery," Gut, vol. 46, no. 5, p. 741, 2000.

[34] G. C. Parkes, J. Brostoff, K. Whelan, and J. D. Sanderson, "Gastrointestinal microbiota in irritable bowel syndrome: their role in its pathogenesis and treatment," The American Journal of Gastroenterology, vol. 103, no. 6, pp. 1557-1567, 2008.

[35] M. Minemura and Y. Shimizu, "Gut microbiota and liver diseases," World Journal of Gastroenterology, vol. 21, no. 6, pp. 1691-1702, 2015.

[36] J. Yoo and S. Kim, "Probiotics and prebiotics: present status and future perspectives on metabolic disorders," Nutrients, vol. 8, no. 3, p. 173, 2016.

[37] E. Distrutti, L. Monaldi, P. Ricci, and S. Fiorucci, "Gut microbiota role in irritable bowel syndrome: new therapeutic strategies," World Journal of Gastroenterology, vol. 22, no. 7, pp. 2219-2241, 2016.

[38] S. K. Mazmanian, C. H. Liu, A. O. Tzianabos, and D. L. Kasper, "An immunomodulatory molecule of symbiotic bacteria directs maturation of the host immune system," Cell, vol. 122, no. 1, pp. 107-118, 2005.

[39] J. L. Round and S. K. Mazmanian, "The gut microbiota shapes intestinal immune responses during health and disease," Nature Reviews Immunology, vol. 9, no. 5, pp. 313-323, 2009.

[40] F. Backhed, H. Ding, T. Wang et al., "The gut microbiota as an environmental factor that regulates fat storage," Proceedings of the National Academy of Sciences, vol. 101, no. 44, pp. 15718-15723, 2004.

[41] E. C. Martens, E. C. Lowe, H. Chiang et al., "Recognition and degradation of plant cell wall polysaccharides by two human gut symbionts," PLoS Biology, vol. 9, no. 12, 2011.

[42] A. Lembo, M. Pimentel, S. S. Rao et al., "Repeat treatment with rifaximin is safe and effective in patients with diarrhea-predominant irritable bowel syndrome," Gastroenterology, vol. 151, no. 6, pp. 1113-1121, 2016.

[43] P. R. Burkholder and I. McVeigh, "Synthesis of vitamins by intestinal bacteria," Proceedings of the National Academy of Sciences, vol. 28, no. 7, pp. 285-289, 1942.

[44] L. G. Albenberg and G. D. Wu, "Diet and the intestinal microbiome: associations, functions, and implications for health and disease," Gastroenterology, vol. 146, no. 6, pp. 1564-1572, 2014.

[45] I. Sekirov, S. L. Russell, L. C. M. Antunes, and B. B. Finlay, "Gut microbiota in health and disease," Physiological Reviews, vol. 90, no. 3, pp. 859-904, 2010. 
[46] E. A. Mayer, K. Tillisch, and A. Gupta, "Gut/brain axis and the microbiota," Journal of Clinical Investigation, vol. 125, no. 3, pp. 926-938, 2015.

[47] J. L. Maupas, P. Champemont, and M. Delforge, “Treatment of irritable bowel syndrome. Double blind trial of Saccharomyces boulardii," Medecine \& Chirurgie Digestives, vol. 12, no. 12, pp. 77-79, 1983.

[48] L. R. Krogsgaard, A. L. Engsbro, C. R. Stensvold, H. V. Nielsen, and P. Bytzer, "The prevalence of intestinal parasites is not greater among individuals with irritable bowel syndrome: a population-based case-control study," Clinical Gastroenterology and Hepatology, vol. 13, no. 3, pp. 507513.e2, 2015.

[49] C. Codling, L. O’Mahony, F. Shanahan, E. M. M. Quigley, and J. R. Marchesi, "A molecular analysis of fecal and mucosal bacterial communities in irritable bowel syndrome," Digestive Diseases and Sciences, vol. 55, no. 2, pp. 392-397, 2010.

[50] I. M. Carroll, Y.-H. Chang, J. Park, R. B. Sartor, and Y. Ringel, "Luminal and mucosal-associated intestinal microbiota in patients with diarrhea-predominant irritable bowel syndrome," Gut Pathogens, vol. 2, no. 1, p. 19, 2010.

[51] Q. Zhou and K. Lu, "Detection of intestinal microbes in patients of IBS with predominant constipation," Modern Diagnosis and Treatment, vol. 24, no. 17, 2013.

[52] T. A. Nelson, S. Holmes, A. V. Alekseyenko et al., "Phylochip microarray analysis reveals altered gastrointestinal microbial communities in a rat model of colonic hypersensitivity," Neurogastroenterology \& Motility, vol. 23, no. 2, 2011.

[53] G. C. Parkes, N. B. Rayment, B. N. Hudspith et al., "Distinct microbial populations exist in the mucosa-associated microbiota of sub-groups of irritable bowel syndrome," Neurogastroenterology \& Motility, vol. 24, no. 1, pp. 31-39, 2012.

[54] I. B. Jeffery, P. W. O'Toole, L. Öhman et al., "An irritable bowel syndrome subtype defined by species-specific alterations in faecal microbiota," Gut, vol. 61, no. 7, pp. 9971006, 2012.

[55] M. Thabane and J. K. Marshall, "Post-infectious irritable bowel syndrome," World Journal of Gastroenterology, vol. 15, no. 29, pp. 3591-3596, 2009.

[56] M. Rajilić-Stojanović, E. Biagi, H. G. H. J. Heilig et al., "Global and deep molecular analysis of microbiota signatures in fecal samples from patients with irritable bowel syndrome," Gastroenterology, vol. 141, no. 5, pp. 1792-1801, 2011.

[57] E. Malinen, T. Rinttila, K. Kajander et al., "Analysis of the fecal microbiota of irritable bowel syndrome patients and healthy controls with real-time PCR," The American Journal of Gastroenterology, vol. 100, no. 2, pp. 373-382, 2005.

[58] E. Malinen, L. Krogius-Kurikka, A. Lyra et al., "Association of symptoms with gastrointestinal microbiota in irritable bowel syndrome," World Journal of Gastroenterology, vol. 16, no. 36, pp. 4532-4540, 2010.

[59] K. McIntosh, D. E. Reed, T. Schneider et al., "FODMAPs alter symptoms and the metabolome of patients with IBS: a randomised controlled trial," Gut, vol. 66, no. 7, pp. 1241-1251, 2017.

[60] R. J. Basseri, S. Weitsman, G. M. Barlow, and M. Pimentel, "Antibiotics for the treatment of irritable bowel syndrome," Gastroenterology and Hepatolology, vol. 7, no. 7, pp. 455493, 2011.
[61] A. Pistiki, I. Galani, E. Pyleris, C. Barbatzas, M. Pimentel, and E. J. Giamarellos-Bourboulis, "In vitro activity of rifaximin against isolates from patients with small intestinal bacterial overgrowth," International Journal of Antimicrobial Agents, vol. 43, no. 3, pp. 236-241, 2014.

[62] M. Pimentel, A. Lembo, W. D. Chey et al., "Rifaximin therapy for patients with irritable bowel syndrome without constipation," New England Journal of Medicine, vol. 364, no. 1, pp. 22-32, 2011.

[63] L. Saha, "Irritable bowel syndrome: pathogenesis, diagnosis, treatment, and evidence-based medicine," World Journal of Gastroenterology: WJG, vol. 20, no. 22, pp. 6759-6773, 2014.

[64] D. Xu, J. Gao, M. Gillilland III et al., "Rifaximin alters intestinal bacteria and prevents stress-induced gut inflammation and visceral hyperalgesia in rats," Gastroenterology, vol. 146, no. 2, pp. 484-496.e4, 2014.

[65] N. B. Kristensen, T. Bryrup, K. H. Allin, T. Nielsen, T. H. Hansen, and O. Pedersen, "Alterations in fecal microbiota composition by probiotic supplementation in healthy adults: a systematic review of randomized controlled trials," Genome Medicine, vol. 8, no. 1, p. 52, 2016.

[66] B. S. Ramakrishna, "Probiotic-induced changes in the intestinal epithelium: implications in gastrointestinal disease," Tropical Gastroenterology, vol. 30, no. 2, pp. 76-85, 2009.

[67] M. Bixquert Jiménez, "Treatment of irritable bowel syndrome with probiotics: an etiopathogenic approach at last?," Revista Espanola De Enfermedades Digestivas, vol. 101, no. 8, pp. 553-564, 2009.

[68] T. Didari, S. Mozaffari, S. Nikfar, and M. Abdollahi, "Effectiveness of probiotics in irritable bowel syndrome: updated systematic review with meta-analysis," World Journal of Gastroenterology, vol. 21, no. 10, pp. 3072-3084, 2015.

[69] D. Compare, A. Rocco, P. Coccoli et al., "Lactobacillus casei $\mathrm{dg}$ and its postbiotic reduce the inflammatory mucosal response: an ex-vivo organ culture model of post-infectious irritable bowel syndrome," BMC Gastroenterology, vol. 17, no. 1, p. 53, 2017.

[70] V. Mezzasalma, E. Manfrini, E. Ferri et al., “A randomized, double-blind, placebo-controlled trial: the efficacy of multispecies probiotic supplementation in alleviating symptoms of irritable bowel syndrome associated with constipation," BioMed Research International, vol. 2016, Article ID 4740907, 10 pages, 2016.

[71] P. H. Johnsen, F. Hilpüsch, J. P. Cavanagh et al., "Faecal microbiota transplantation versus placebo for moderate-tosevere irritable bowel syndrome: a double-blind, randomised, placebo-controlled, parallel-group, single-centre trial," The Lancet Gastroenterology \& Hepatology, vol. 3, no. 1, pp. 1724, 2018.

[72] S. I. Halkjær, A. H. Christensen, B. Z. S. Lo et al., "Faecal microbiota transplantation alters gut microbiota in patients with irritable bowel syndrome: results from a randomised, double-blind placebo-controlled study," Gut, vol. 67, no. 12, pp. 2107-2115, 2018.

[73] G. Ianiro, M. Maida, J. Burisch et al., "Efficacy of different faecal microbiota transplantation protocols for Clostridium difficileinfection: a systematic review and meta-analysis," United European Gastroenterology Journal, vol. 6, no. 8, pp. 1232-1244, 2018.

[74] S. Gupta, E. Allen-Vercoe, and E. O. Petrof, "Fecal microbiota transplantation: in perspective," Therapeutic Advances in Gastroenterology, vol. 9, no. 2, pp. 229-239, 2015. 
[75] H. H. Kernsten, "Intentional replacement of bacteria following antibiotic therapy," Journal Iowa State Medical Society, vol. 48, no. 5, pp. 240-243, 1958.

[76] B. Eiseman, W. Silen, G. S. Bascom, and A. J. Kauvar, "Fecal enema as an adjunct in the treatment of pseudomembranous enterocolitis," Surgery, vol. 44, no. 5, pp. 854-859, 1958.

[77] G. Cammarota, G. Ianiro, H. Tilg et al., "European consensus conference on faecal microbiota transplantation in clinical practice," Gut, vol. 66, no. 4, pp. 569-580, 2017.

[78] E. Allen-Vercoe, G. Reid, N. Viner et al., "A Canadian Working Group report on fecal microbial therapy: microbial ecosystems therapeutics," Canadian Journal of Gastroenterology, vol. 26, no. 7, pp. 457-462, 2012.

[79] C. R. Kelly, S. Kahn, P. Kashyap et al., "Update on fecal microbiota transplantation 2015: indications, methodologies, mechanisms, and outlook," Gastroenterology, vol. 149, no. 1, pp. 223-237, 2015.

[80] C. Owens, E. Broussard, and C. Surawicz, "Fecal microbiota transplantation and donor standardization," Trends in Microbiology, vol. 21, no. 9, pp. 443-445, 2013.

[81] B. Cui, Q. Feng, H. Wang et al., "Fecal microbiota transplantation through mid-gut for refractory Crohn's disease: safety, feasibility, and efficacy trial results," Journal of Gastroenterology and Hepatology, vol. 30, no. 1, pp. 51-58, 2015.

[82] B. Cui, P. Li, L. Xu et al., "Step-up fecal microbiota transplantation strategy: a pilot study for steroid-dependent ulcerative colitis," Journal of Translational Medicine, vol. 13, no. 1, 2015.

[83] E. Gough, H. Shaikh, and A. R. Manges, "Systematic review of intestinal microbiota transplantation (fecal bacteriotherapy) for recurrent Clostridium difficile infection," Clinical Infectious Diseases, vol. 53, no. 10, pp. 994-1002, 2011.

[84] J. König, A. Siebenhaar, C. Högenauer et al., "Consensus report: faecal microbiota transfer-clinical applications and procedures," Alimentary Pharmacology \& Therapeutics, vol. 45, no. 2, pp. 222-239, 2017.

[85] Z. D. Jiang, N. J. Ajami, J. F. Petrosino et al., "Randomised clinical trial: faecal microbiota transplantation for recurrent Clostridum difficile infection - fresh, or frozen, or lyophilised microbiota from a small pool of healthy donors delivered by colonoscopy," Alimentary Pharmacology \& Therapeutics, vol. 45, no. 7, pp. 899-908, 2017.

[86] A. Khoruts, J. Dicksved, J. K. Jansson, and M. J. Sadowsky, "Changes in the composition of the human fecal microbiome after bacteriotherapy for recurrent Clostridium difficileassociated diarrhea," Journal of Clinical Gastroenterology, vol. 44, no. 5, pp. 354-360, 2010.

[87] L. J. Brandt and O. C. Aroniadis, "An overview of fecal microbiota transplantation: techniques, indications, and outcomes," Gastrointestinal Endoscopy, vol. 78, no. 2, pp. 240249, 2013.

[88] O. C. Aroniadis and L. J. Brandt, "Intestinal microbiota and the efficacy of fecal microbiota transplantation in gastrointestinal disease," Gastroenterology \& Hepatology, vol. 10, no. 4, 2014.

[89] R. McEvoy, "Bowel-flora alteration: a potential cure for inflammatory bowel disease and irritable bowel syndrome?," Medical Journal of Australia, vol. 151, no. 2, pp. 112-112, 1989.

[90] D. Pinn, O. Aroniadis, and L. Brandt, "Follow-up study of fecal microbiota transplantation (FMT) for the treatment of refractory irritable bowel syndrome (IBS)," American Journal Of Gastroenterology, vol. 108, p. S563, 2013.

[91] T. Mazzawi, M. El-Sahy, G. Lied, O. H. Gilja, J. G. Hatlebakk, and T. Hausken, "Effect of faecal microbiota transplantation on the symptoms and duodenal enteroendocrine cells in patients with irritable bowel syndrome," United European Gastroenterology Journal, vol. 4, 2016.

[92] E. V. Gaidar, M. M. Kostik, M. F. Dubko, V. V. Masalova, L. S. Snegireva, and E. A. Isupova, The Efficiency of Adalimumab in Cases of Chronic Methotrexate-Resistant Juvenile Idiopathic Arthritis-Associated Anterior Uveitis: Retrospective Case Series Study, Pediatric Pharmacology, 2016.

[93] A. C. Ford, "Stool as a treatment for IBS: more questions than answers?," The Lancet Gastroenterology \& Hepatology, vol. 3, no. 1, pp. 2-3, 2018.

[94] T. Holvoet, M. Joossens, B. Jerina et al., "617 - fecal microbiota transplantation in irritable bowel syndrome with predominant abdominal bloating: results from a double blind, placebo-controlled clinical trial," Gastroenterology, vol. 154, no. 6, pp. S-130, 2018.

[95] S. Holster, C. M. Lindqvist, D. Repsilber et al., "The effect of allogenic versus autologous fecal microbiota transfer on symptoms, visceral perception and fecal and mucosal microbiota in irritable bowel syndrome," Clinical and Translational Gastroenterology, vol. 10, no. 4, p. e00034, 2019.

[96] O. C. Aroniadis, L. J. Brandt, C. Oneto et al., "742 - a doubleblind, randomized, placebo-controlled trial of fecal microbiota transplantation capsules (FMTC) for the treatment of diarrhea-predominant irritable bowel syndrome (IBS-D)," Gastroenterology, vol. 154, no. 6, pp. S-154-S-155, 2018.

[97] O. C. Aroniadis, L. J. Brandt, C. Oneto et al., "Faecal microbiota transplantation for diarrhoea-predominant irritable bowel syndrome: a double-blind, randomised, placebocontrolled trial," The Lancet Gastroenterology \& Hepatology, vol. 4, no. 9, pp. 675-685, 2019.

[98] E. Shah and M. Pimentel, "Placebo effect in clinical trial design for irritable bowel syndrome," Journal of neurogastroenterology and motility, vol. 20, no. 2, pp. 163-170, 2014.

[99] M. Baxter and A. Colville, "Adverse events in faecal microbiota transplant: a review of the literature," Journal of Hospital Infection, vol. 92, no. 2, pp. 117-127, 2016.

[100] R. E. Ooijevaar, E. M. Terveer, H. W. Verspaget, E. J. Kuijper, and J. J. Keller, "Clinical application and potential of fecal microbiota transplantation," Annual Review of Medicine, vol. 70, no. 1, pp. 335-351, 2019. 


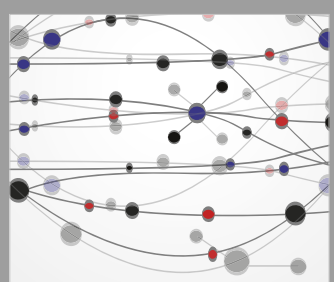

The Scientific World Journal
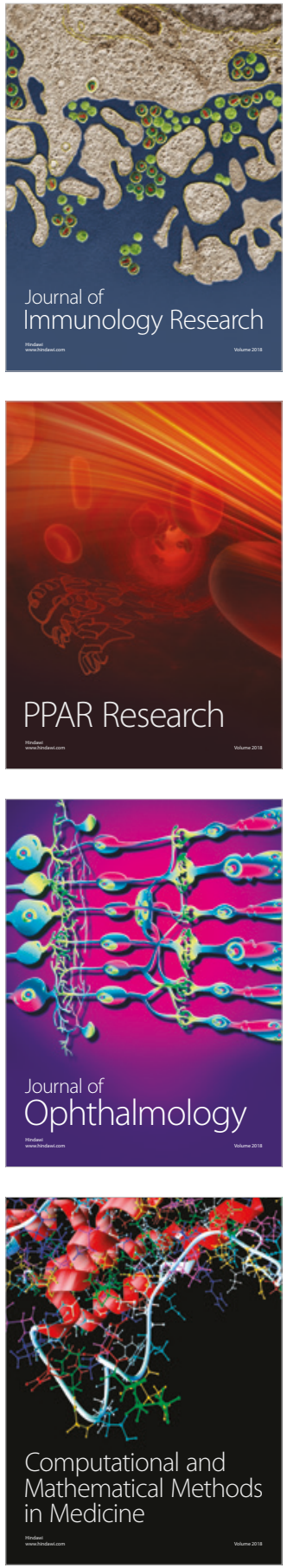



Gastroenterology Research and Practice

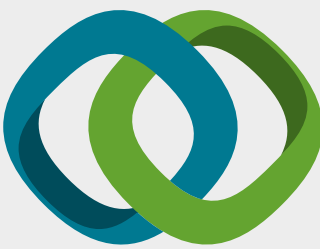

\section{Hindawi}

Submit your manuscripts at

www.hindawi.com


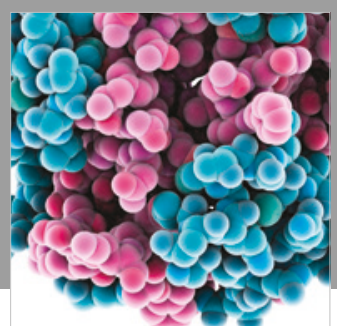

ournal of

Diabetes Research

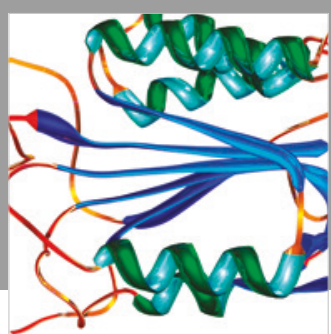

Disease Markers
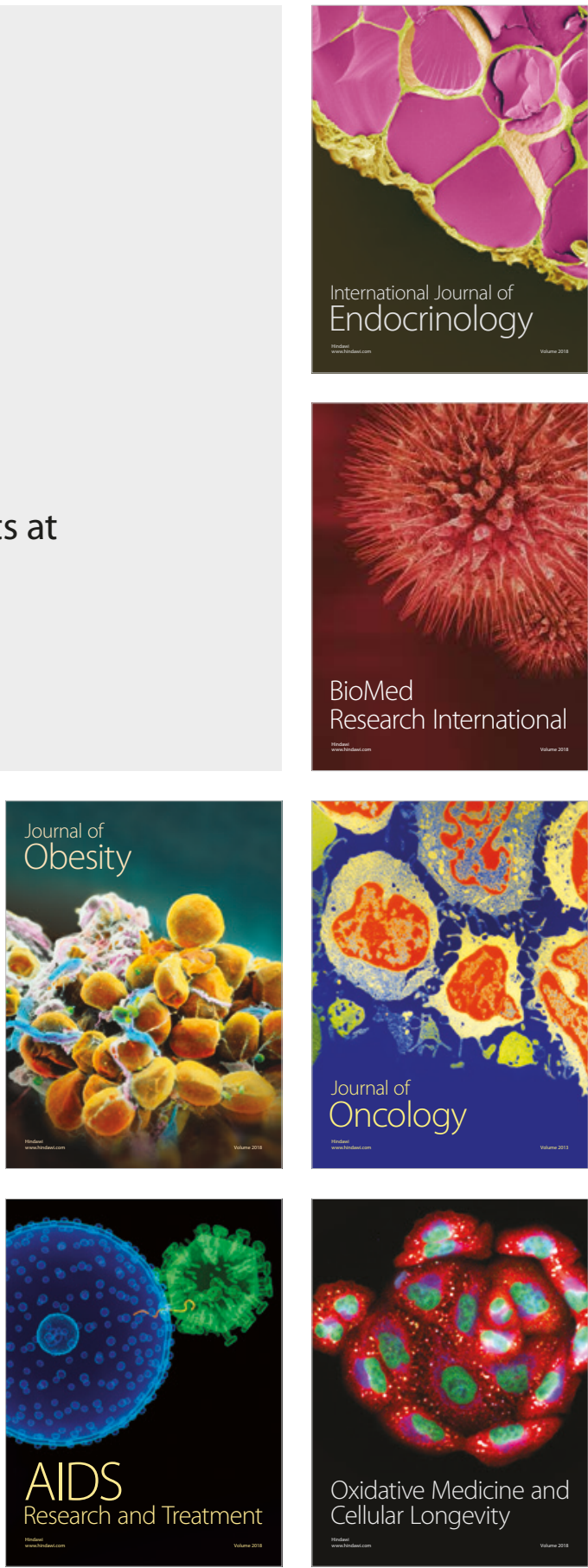